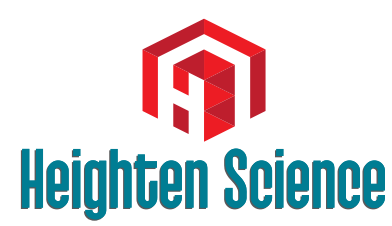

P U B L I C I T I O N S Corporation

\title{
A new heart: portraying the physiologic anatomo-functional reconstruction in ischemic cardiomyopathy
}

\author{
Marco Cirillo ${ }^{1 *}$, Marco Campana ${ }^{2}$, Anna Bressanelli ${ }^{3}$ and \\ Giovanni Troise ${ }^{4}$ \\ ${ }^{1}$ Heart Failure Surgery Unit, Poliambulanza Foundation Hospital, Brescia, Italy \\ ${ }^{2}$ Echocardiography Laboratory, Cardiology Unit, Cardiovascular Department, Poliambulanza Foundation \\ Hospital, Brescia, Italy \\ ${ }^{3}$ Radiology Department, Poliambulanza Foundation Hospital, Brescia, Italy \\ ${ }^{4}$ Cardiac Surgery Unit, Cardiovascular Department, Poliambulanza Foundation Hospital, Brescia, Italy
}

*Address for Correspondence: Marco Cirillo, Heart Failure Surgery Unit, Poliambulanza Foundation HospitalVia Leonida Bissolati 57, 25125 Brescia Italy, Tel: +39030 351 8534; Fax: +39030351 5244; Email: marco.cirillo@poliambulanza.it

Submitted: 17 August 2017

Approved: 14 September 2017

Published: 15 September 2017

Copyright: @ 2017 Cirillo M, et al. This is an open access article distributed under the Creative Commons Attribution License, which permits unrestricted use, distribution, and reproduction in any medium, provided the original work is properly cited.

\section{Summary}

Fiber-based model of the left ventricle is known since 1628 but the complex 3D structure of myocardial fibers has not taken into account in normalcy or in disease until the last decade. We here present the case of a 60-year-old female patient affected by ischemic cardiomyopathy and severe left ventricular dysfunction. Left ventricle was reconstructed according to a novel surgical technique aimed at rebuilding an elliptical ventricular chamber and redirecting myocardial bundles of fibers in a near-normal orientation, by means of an original suturing technique. Left ventricular torsion was restored, proving the reorientation of myocardial fibres' bundles. The restored physiologic shape was maintained along the years, gradually improving global ejection fraction and diastolic indices, showing a positive remodeling induced by the optimised geometrical and functional parameters.

The unexpected and never proven before renewal of ventricular torsion is an adjunctive element of ventricular efficiency, mainly in ventricles that work at a critical mechanics. A new fiber-based reading of heart function could improve clinical and functional outcomes and address some unsolved issues in the surgical treatment of ischemic cardiomyopathy as well as in medical approaches to the diseased myocardium.

\section{Introduction}

Fiber-based model of the left ventricle is known since 1628, when William Harvey described it in his "Exercitatio Anatomica de Motu Cordis et Sanguinis in Animalibus", but the complex 3D structure of interlaced, gradually-oriented myocardial fibers has not taken into account in normalcy or in disease until the last decade. This renewed interest in the true myocardial structure was driven and promoted by new imaging techniques, like 2D speckle tracking echocardiography. At the same time the tight dependency between structure and function was progressively considered also in the field of surgical reconstruction of the left ventricle in ischemic cardiomyopathy. A slow but constant awareness is growing that some unsuccessful or limited techniques and studies could be attributed to the lack of consideration of the intrinsic anatomofunctional structure of the myocardium. Best clinical results have been obtained if all of the key structural, geometric and functional characteristics of the heart were restored, keeping in mind its normal anatomy [1].

A new heart, or better a new vision of the heart should be considered to achieve positive results both in medical and surgical approaches to the diseased myocardium.

\section{Case Report and Imaging}

We here present the case of a 60 -year-old female patient affected by ischemic 
cardiomyopathy and severe left ventricular dysfunction. She had the main episode of myocardial infarction 24 months before the surgical correction. Mitral regurgitation was absent. Left ventricle was reconstructed according to a novel surgical technique aimed at rebuilding an elliptical ventricular chamber with a new apex and redirecting myocardial bundles of fibers, displaced by the infarct scar, in a near-normal orientation, by means of an original suturing technique $[2,3]$. This technique is based on the evidence that transmural courses of fibers' orientation angles near infarct zones were similar to those of normal myocardium [4] and on anatomical map of myocardial fibers' disposition, common to all mammals.

Echocardiographic data of five control visits (preoperative, 6 days after the operation, 4 months and 2 and 4 years follow-up) are resumed in table 1 . The physiologically reconstructed ventricle is represented by means of echocardiographic (Figure 1 and 4; Video 1 and 5) and magnetic resonance (Figure 2 and 3; Videos 2, 3 and 4) imaging.

\begin{tabular}{|c|c|c|c|c|c|}
\hline & \multicolumn{5}{|c|}{ Echocardiographic data } \\
\hline & Preop & Postop 6 days & 4 months & 2 years & 4 years \\
\hline EDV & 221 & 81 & 87 & 109 & 128 \\
\hline EDVi & 127 & 51 & 50 & 63 & 74 \\
\hline ESV & 173 & 42 & 46 & 61 & 67 \\
\hline ESVi & 99 & 27 & 26 & 32 & 34 \\
\hline $\mathrm{EF}$ & 22 & 48 & 47 & 44 & 47 \\
\hline$E / A$ & 0.68 & 1.0 & 0.9 & 1.0 & 0.6 \\
\hline DT & 194 & 167 & 243 & 115 & 262 \\
\hline APROT & -4.13 & 4.6 & & & 1.03 \\
\hline TORS & 2.0 & 7.0 & & & 3.95 \\
\hline
\end{tabular}

Abbreviations: EDV, end-diastolic volume, $\mathrm{ml}$; EDVI, end-diastolic volume index, $\mathrm{ml} / \mathrm{m}^{2}$; ESV, end-systolic volume, $\mathrm{ml}$; ESVI, end-systolic volume index, $\mathrm{ml} / \mathrm{m}^{2}$; EF, ejection fraction, \%; E/A, E/A ratio; DT, E-wave deceleration time, $\mathrm{ms}$; APROT, apical rotation, degrees; TORS, LV torsion, degrees.
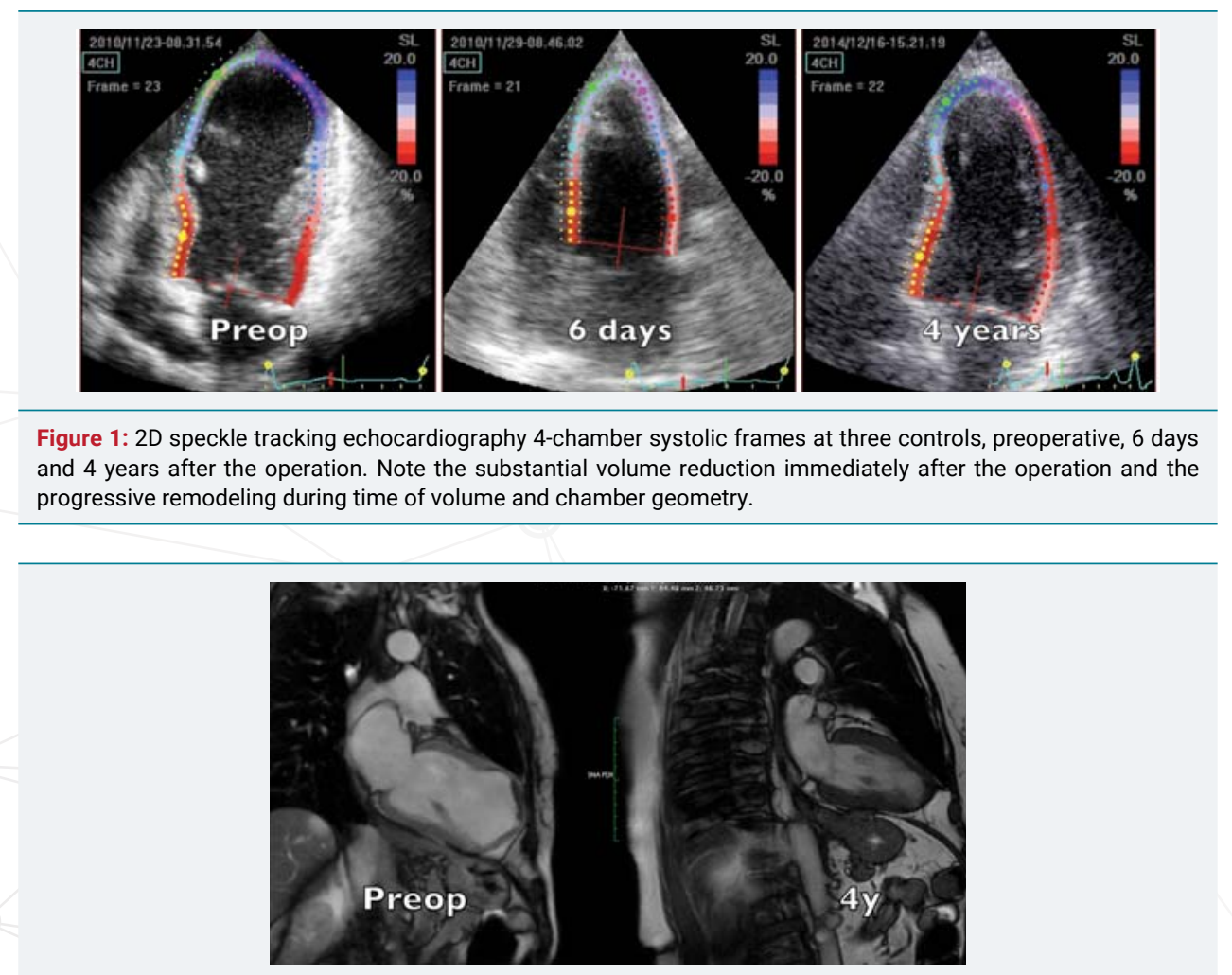

Figure 2: Magnetic resonance long axis systolic frames at two controls, preoperative and 4 years after the operation. A near-normal chamber geometry and wall thickening is evident. Note the reduction of left atrial volume, even in the absence of preoperative mitral regurgitation. 


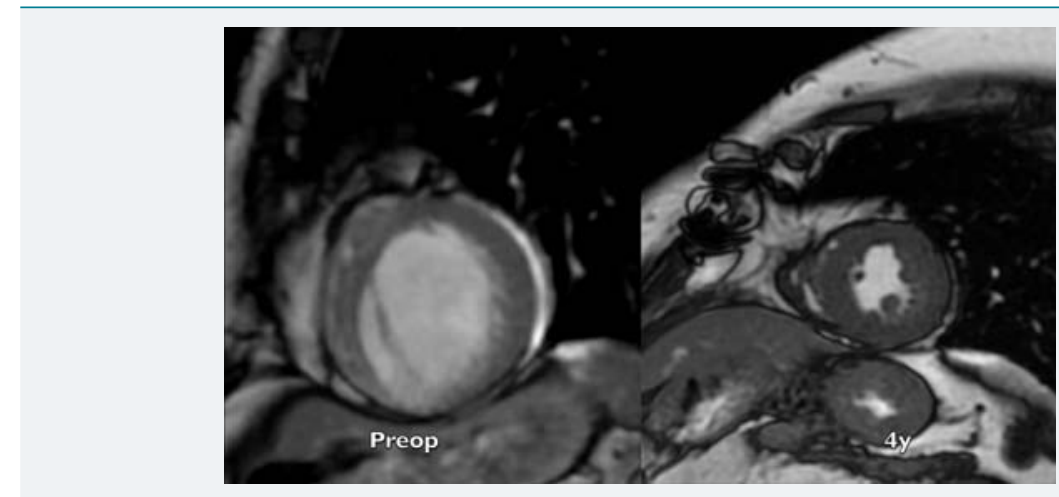

Figure 3: Magnetic resonance short axis systolic frames at two controls, preoperative and 4 years after the operation. The whole left ventricular chamber is restored and the remote myocardium regains its mechanics.

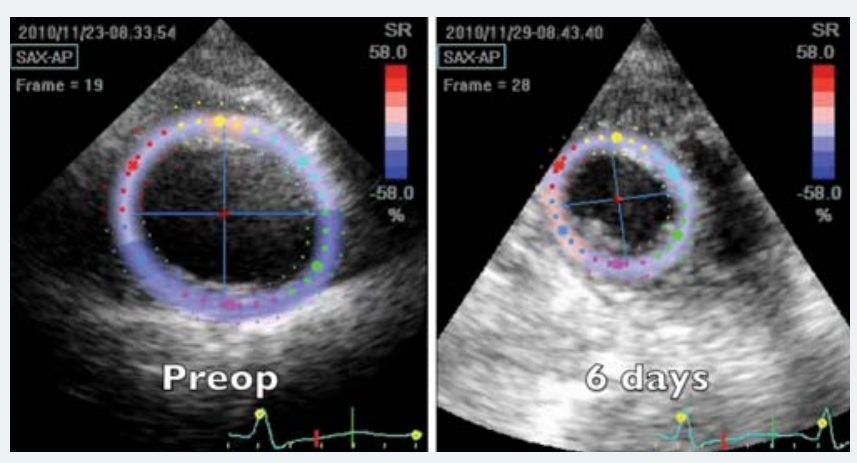

Figure 4: 2D speckle tracking echocardiography short axis apical systolic frames at two controls, preoperative and 6 days after the operation.

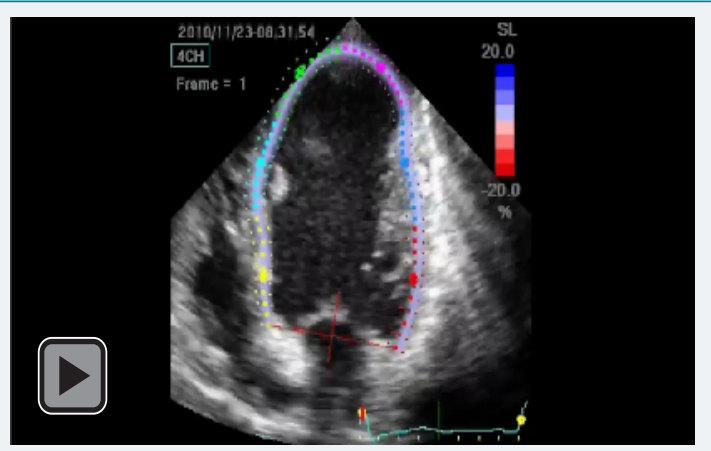

Video 1: Echocardiography apical four-chamber view; comparison between preoperative, 6 days, and 4 years after the operation controls. Note the substantial volume reduction immediately after the operation and the progressive, positive adjustment during time of volume and chamber geometry, promoted by structural and geometric physiological reconstruction.

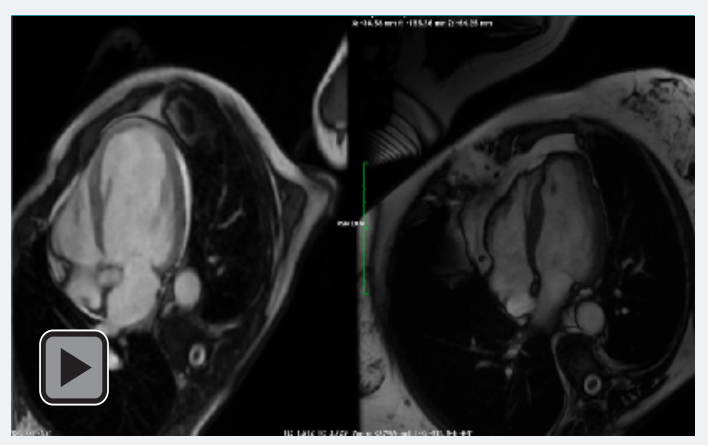

Video 2: Magnetic resonance four-chamber view, comparison between preoperative and 4 years controls. 


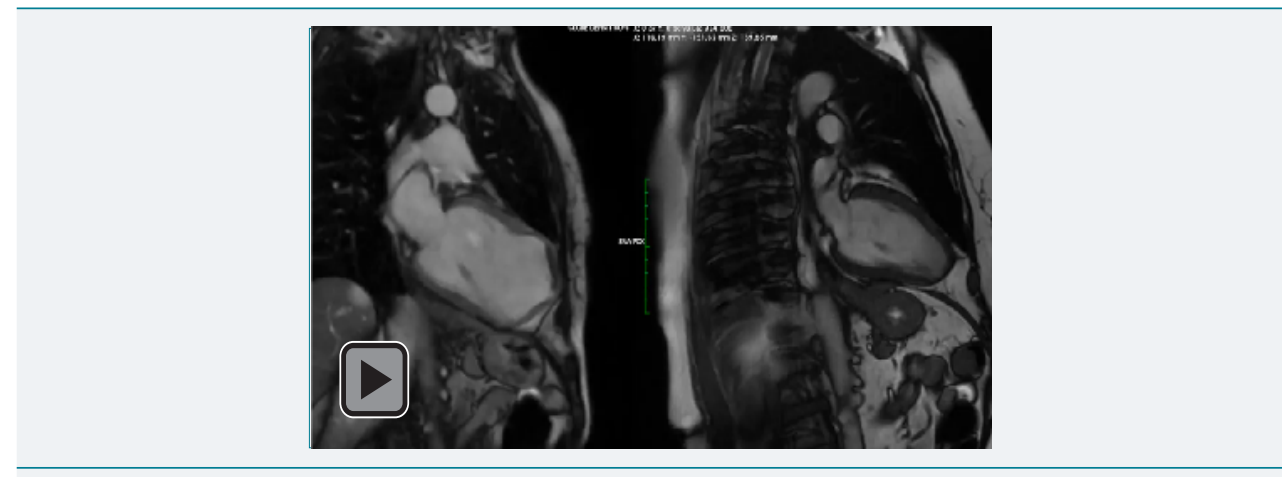

Video 3: Magnetic resonance long axis view, comparison between preoperative and 4 years controls.

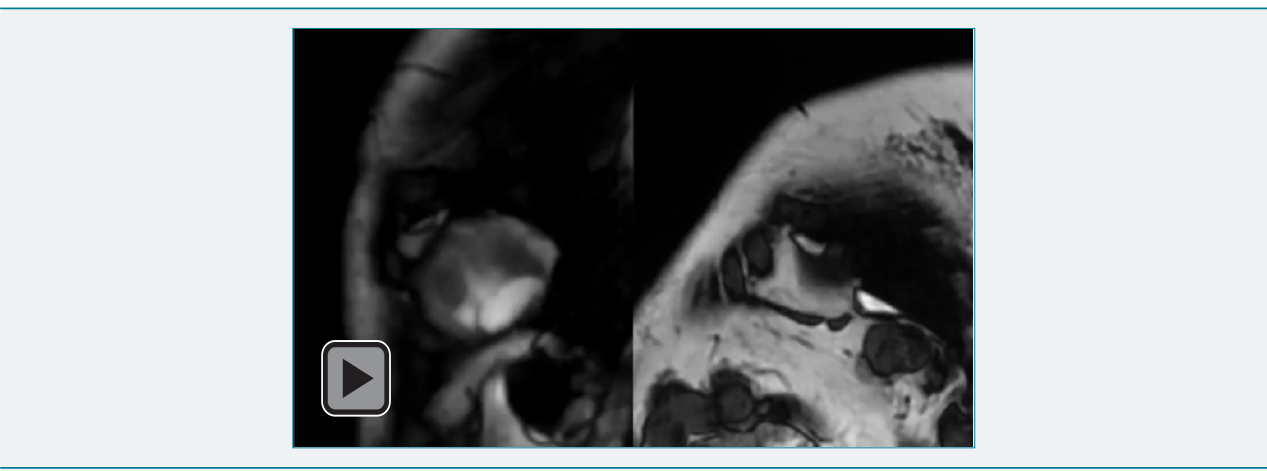

Video 4: Magnetic resonance short axis views, comparison between preoperative and 4 years controls. Note the evident apical and chamber reconstruction, and the remarkable improvement of global left ventricular systolic thickening and diastolic relaxation.

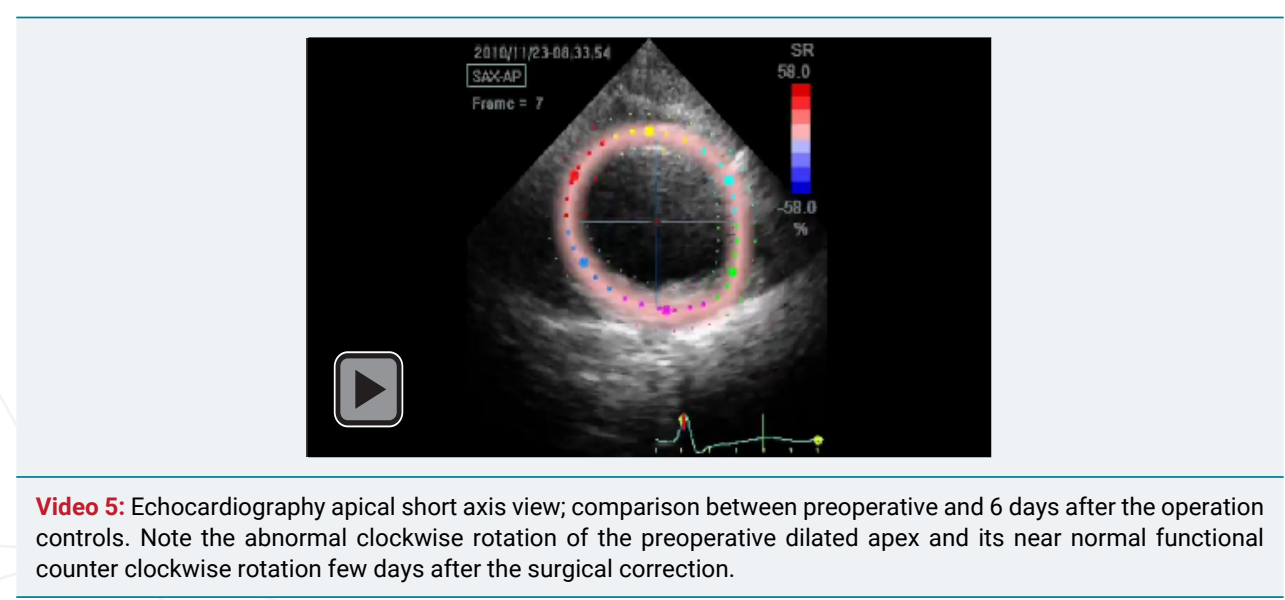

Preoperative imaging shows a very large dyskinetic region equal to around half of the ventricular chamber, conditioning a box-shaped ventricle with severely impaired global contraction, wall thickening, and diastolic function. Surgical reconstruction achieved an elliptic chamber with a normal volume and a new apex that were maintained along the years, gradually improving global ejection fraction and diastolic indices (Table 1), showing a positive remodeling induced by the optimised geometrical and functional parameters, left ventricular torsion included. Remote regions show a renewed, increased thickening granted by the better chamber geometry and fibers' realignment, and the whole left ventricle shows a time-dependent restoration of its functional reserve, preoperatively hidden by the unfavourable functional conditions.

Apical region study (Figure 4 and Video 5) clearly demonstrates an improvement in apical geometry and rotation (from - 4.1 degrees to 4.6 degrees) in the immediate postoperative period induced by the surgically reoriented bundles of fibers. Consequently, left ventricular torsion improved to 7 degrees after the operation, with 
a reduction to about 4 degrees four years after surgery, due to a new-onset small apical dyskinetic area that reduces counterclockwise rotation to 1.3 degrees. Magnetic resonance short axis views (Figure 3 and Video 4) highlight the physiologically restored left ventricle along its whole length, from the apex to the base. The key surgical steps of this technique are: a) the septal rim of the suture is as long as the patch; b) the lateral rim of the suture shrinks the displaced fibers to a more physiologic orientation, given that it must adapt the dilated lateral wall onto the patch; c) a new apex is rebuilt (elliptic shape); d) a very small "akinetic" patch is used to bring closer the normal myocardium, redirecting fibers in a more physiologic disposition (Video 6).

\section{Conclusions and Perspective}

Surgical ventricular reconstruction should achieve the full spectrum of recoverable physiological parameters to regain functional potentiality of remote myocardium. The unexpected and never proven before renewal of apical rotation and ventricular torsion is an adjunctive element of ventricular efficiency, mainly in ventricles that work at a critical mechanics and could favour the shown positive remodeling during time. Longterm results of this surgical technique have been recently validated [5]. A renewed fiber-based reading of heart function could improve clinical and functional outcomes, address some unsolved issues in the surgical treatment of ischemic cardiomyopathy and be a basic science common denominator for all heart diseases.

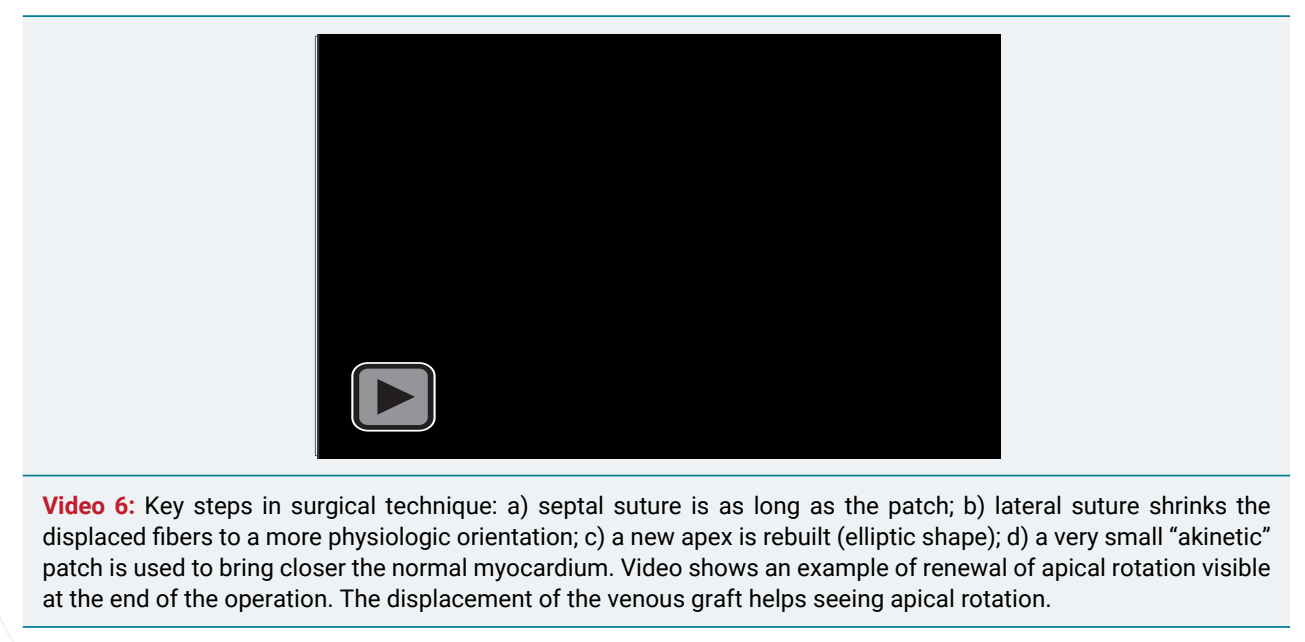

\section{References}

1. Isomura T, Horii T, Suma H, Buckberg GD, RESTORE Group. Septal anterior ventricular exclusion operation (Pacopexy) for ischemic dilated cardiomyopathy: treat form not disease. Eur J Cardiothorac Surg. 2006; 29: 245-250. Ref.: https://goo.gl/2RXXHf

2. Cirillo $M$, Arpesella $G$. Rewind the heart: a novel technique to reset heart fibers' orientation in surgery for ischemic cardiomyopathy. Med Hypotheses. 2008; 70: 848-854. Ref.: https://goo.gl/Mvz3KH

3. Cirillo M. A new surgical ventricular restoration technique to reset residual myocardium's fiber orientation: the "KISS" procedure. Ann Surg Innov Res. 2009; 3: 6. Ref.: https://goo.gl/PwzDpD

4. Chen J, Song S, Liu W, McLean M, Allen JS, et al. Remodeling of cardiac fiber structure after infarction in rats quantified with diffusion tensor MRI. Am J Physiol Heart Circ Physiol. 2003; 285: 946-954. Ref.: https://goo.gl/gao5HM

5. Cirillo M, Campana M, Brunelli F, Dalla Tomba M, Mhagna Z, et al. Time series analysis of physiological left ventricular reconstruction in ischemic cardiomyopathy. J Thorac Cardiovasc Surg 2016; 152: 382-391. Ref.: https://goo.gl/ZMDBGy 